\section{Safely Chemotherapy Administration in Patient with Small-cell Lung Cancer and End-stage Renal Disease Undergoing Haemodialysis}

\author{
Daliborka Bursac ${ }^{1,2^{*}}$ \\ ${ }^{1}$ Department for Chemotherapy, Institute for Pulmonary Diseases of Vojvodina, Sremska Kamenica, Serbia
}

${ }^{2}$ Faculty of Medicine Novi Sad, University of Novi Sad, Serbia

*Corresponding author: Daliborka Bursac, Faculty of Medicine Novi Sad, Department for Chemotherapy, Institute for Pulmonary Diseases of Vojvodina, Sremska Kamenica, Serbia, Tel: +381642839713; E-mail: dadabursac@gmail.com; daliborka.bursac@mf.uns.ac.rs

Received date: August 27, 2018; Accepted date: October 25, 2018; Published date: October 31, 2018

Copyright: $\odot 2018$ Bursac D. This is an open-access article distributed under the terms of the Creative Commons Attribution License, which permits unrestricted use, distribution, and reproduction in any medium, provided the original author and source are credited.

Keywords: Small-cell lung cancer; Chemotherapy; Haemodialysis

\section{Introduction}

Lung cancer is the leading cause of death in many countries. Smallcell lung cancer is chemio-sensitive malignant disease and the application of chemotherapy, usually, is the first and most important therapeutic approach. However, there are no clear recommendations for the use of chemotherapy in the presence of chronic renal failure treated with haemodialysis. The case of a 54-year-old patient with small-cell lung cancer and chronic renal failure due to hypertensive nephropathy on haemodialysis is presented. As a first line chemotherapy, 6 cycles of carboplatin/etoposide chemotherapy were administered. After disease progression, second line chemotherapy with 4 cycles cyclophosphamide/vincristine/doxorubicin was applied safely.

\section{Case Report}

A 54-year-old man with chronic renal failure due to hypertensive nephropathy presented with symptoms dispnea, cough and hoarseness in March 2014. He had been undergoing haemodialysis three times a week for the past 5 years. The patient was a smoker with a 40 pack-peryear history. Physical examination has established an enlarged lymph node in the left supraclavicular region. His Eastern Cooperative Oncology Group (ECOG) performance status was 1. In the blood laboratory analyses creatinine $(769 \mu \mathrm{mol} / \mathrm{L})$ and urea $(12.9 \mathrm{mmol} / \mathrm{L})$ were elevated with anaemia (erythrocytes $3.2 \times 10^{12} / \mathrm{L}$, haemoglobin $113 \mathrm{~g} / \mathrm{L}$ ). Arterial blood gas values were without a pathological finding. Chest X-ray showed left upper lobar infiltration with hilar enlargement. On CT scan of the chest, tumour infiltration in the left upper lobe, 64 $\mathrm{mm}$ in diameter was noticed with enlarged lymph nodes left paratracheal, diameter $55 \mathrm{~mm}$ and subcarinal $45 \mathrm{~mm}$, as well as nodule in the lingula of $25 \mathrm{~mm}$ diameter (Figure 1). On the CT scan of the abdomen an increase in retroperitoneal lymph nodes of $28 \mathrm{~mm}$ was observed.

Supraclavicular lymph node biopsy confirmed small-cell lung cancer, with the clinical and radiological stage of the disease T3N3M1b (extensive stage, extensive diseases-ED). In accordance with his good performance status and chemiosensitivity of the disease we decided for carboplatin/etoposide chemotherapy. First-line chemotherapy in 6 cycles was applied every 4 weeks. Carboplatin was applied on the first day of the cycle, which was also an inter-dialysis day. The dose was calculated according to the Calvert formula [Dose $=\mathrm{AUC}(\mathrm{GFR}+25)]$.
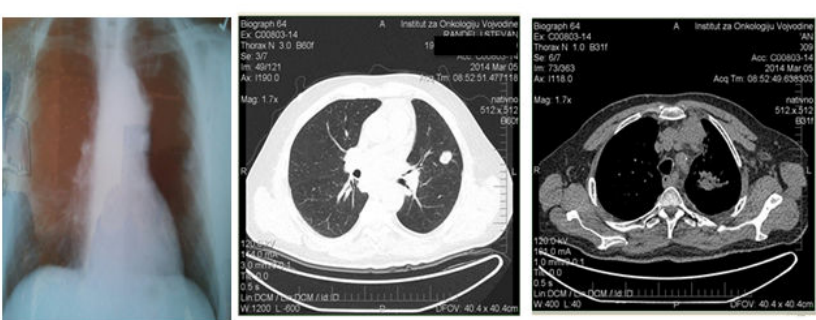

Figure 1: Chest $\mathrm{x}$-ray and CT before carboplatin/etoposide chemotherapy.

Etoposide is applied to 2, 3, and day 4 of the cycle (dialysis days), in a $50 \%$ reduced dose $\left(50 \mathrm{mg} / \mathrm{m}^{2}\right)$. Haemodialysis was performed 3 times a week (Figure 2).

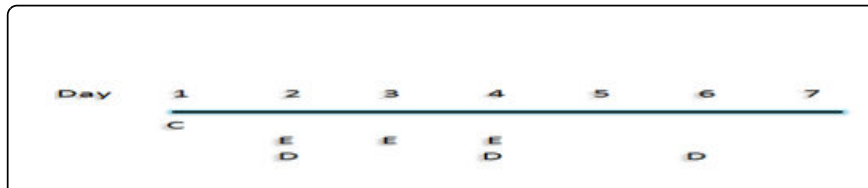

Figure 2: Review of the application first- line chemotherapy and haemodialysis.

During the first-line chemotherapy, haematological toxicities occurred (grade-2 anaemia, grade-3 neutropenia and grade-2 thrombocytopenia) successfully treated using granulocyte-colony stimulating factor (G-CSF) and transfusion of blood products.

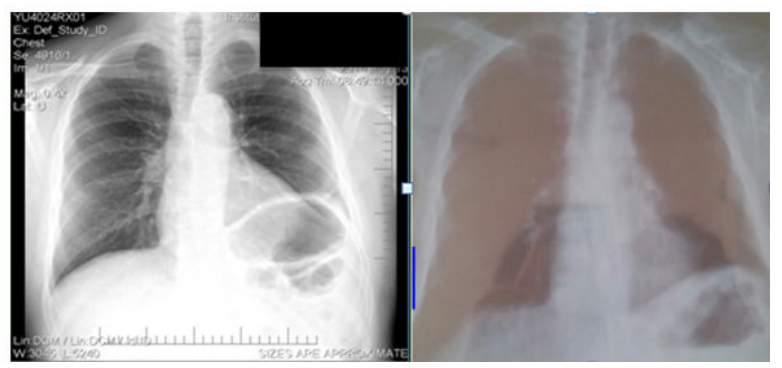

Figure 3: Chest $\mathrm{x}$-ray one and two months after first-line chemotherapy. 
On the first control examination that followed a month after the application of chemotherapy, the patient achieved a partial radiological response and he was in good general condition, ECOG performance status 0. But, after only one month, in October 2014, radiological progression on chest X-ray was detected with left supraclavicular lymphadenopathy by physical examination (Figure 3). Cyclophosphamide/Adriamycin (doxorubicin)/vincristine (CAV) protocol as a second-line chemotherapy was indicated.

Patient received four cycles of CAV chemotherapy at 3 week intervals. Cyclophosphamide administered in a dose reduced by $25 \%$ of the predicted dose of $750 \mathrm{mg} / \mathrm{m}^{2}$, doxorubicin at a dose of $40 \mathrm{mg} / \mathrm{m}^{2}$ and vincristine at a dose of $1.3 \mathrm{mg} / \mathrm{m}^{2}$. All drugs were administered on the inter-dialysis day, and haemodialysis was performed 3 times a week (Figure 4).

\begin{tabular}{|c|c|c|c|c|c|c|c|}
\hline Day & 1 & 2 & 3 & 4 & 5 & 6 & 7 \\
\hline & $\begin{array}{l}\text { C } \\
\text { A }\end{array}$ & & & & & & \\
\hline
\end{tabular}

Figure 4: Review of the application second-line chemotherapy and haemodialysis.

The control chest CT examination, three months after second line chemotherapy and one year after initial diagnosis, showed a tumor in the left upper lobe of $53 \mathrm{~mm}$ diameter, with enlarged lymph nodes left paratracheal $55 \mathrm{~mm}$, subcarinal $45 \mathrm{~mm}$ and nodule in lingula $24 \mathrm{~mm}$, which according to RECIST (Response Evaluation Criteria In Solid Tumours v 1.1) criteria corresponded to stable disease-SD (Figure 5).
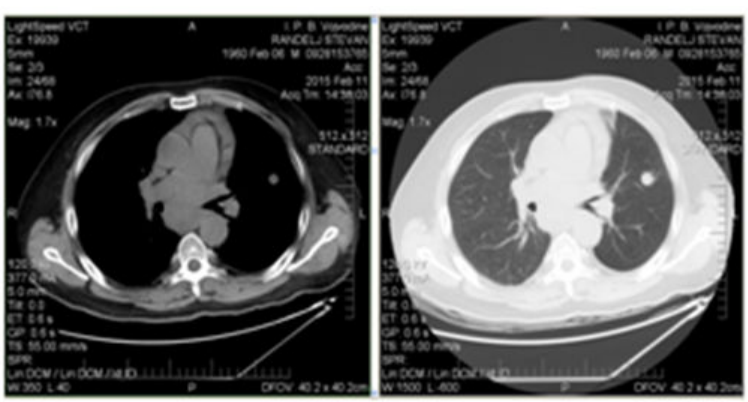

Figure 5: Chest CT after second-line chemotherapy.

\section{Discussion}

In modern oncology, it is not uncommon that patients with chronic renal insufficiency at hemodialysis have malignant disease at the same time. The safety and efficacy of chemotherapy in these patients is not clearly defined. The presence of an end-stage renal disease requiring hemodialysis in patients with malignant disease is a bad prognostic sign. These patients have been excluded from clinical trials and it is not possible to establish recommendations for the application of chemotherapy $[1,2]$.

In dialysis patients, two moments are important;

-Drug dosage reduction may be required to avoid over dosage and drug toxicity,
- Applications of chemotherapy and haemodialysis should be wellcoordinated. It is necessary to determine what fraction of drugs is removed by haemodialysis. For drugs with high renal elimination chemotherapy should be administered after dialysis to avoid drug removal and loss of efficacy. However, for drugs that are not significantly removed by haemodialysis, administration can be carried out anytime [3].

Data on the application of chemotherapy in patients undergoing haemodialysis are deficient, mostly case reports or trials on a small group of patients.

Kim et al. reported the pharmacokinetics of carboplatin and etoposide in patients with lung cancer and chronic renal failure undergoing haemodialysis. The paper has shown that plasma chemotherapeutic values are similar in patients with normal renal function and in those with renal insufficiency treated with haemodialysis. Carboplatin concentrations are rapidly decreasing in the first $24 \mathrm{~h}$, but remain longer in plasma during subsequent chemotherapy cycles. Concentration of etoposide in plasma has a slow decline, but also persists longer until the next cycle of chemotherapy [2]. Janus et al. recommended the application of certain chemotherapeutics in haemodialysis patients [3]. Carboplatin is eliminated by more than $95 \%$ of the kidneys and is recommended to be applied on an inter-dialysis day. About $70 \%-80 \%$ of the administered dose of cyclophosphamide is transformed into metabolites by hepatic enzymes and $30 \%-60 \%$ is eliminated by the kidneys. Recommendations are to reduce the dose by $25 \%$ in haemodialysis patients.

Data on the pharmacokinetics of doxorubicin in patients with renal failure are very limited. Doxorubicin is not eliminated by the urinary tract, and it is not necessary modified dose. About $40 \%$ of the dose of etoposide is eliminated by the kidneys. It is recommended to reduce the dose by $50 \%$ in haemodialysis patients to avoid accumulation of the drug and the toxic effects. As etoposide is not removed by haemodialysis can be used before or after haemodialysis sessions [3].

Recommendations are certainly to continue with pharmacokinetic drug testing in haemodialysis patients, because only this way can determine the degree of drug elimination, safety and efficacy of chemotherapy [4-8].

Management of patients with end-stage renal disease undergoing chemotherapy is difficult, so a better risk/benefit ratio should be considered. The prognosis of dialysed cancer patients is poor compared with that of non-dialysed patients. When selecting patients it is important to consider the goal of chemotherapy ('adjuvant', 'curative' or 'palliative'), the tumour-related and the renal diseaserelated life expectancy, the impact of chemotherapy on the quality of life and patient characteristics (age, performance status and comorbidities) [7]. Small-cell lung cancer is an extremely aggressive disease and the basis of treatment is the application of chemotherapy. In the absence of general recommendations for the treatment of these patients, we consider that it is necessary to analyse the case of each patient individually taking into account the above factors.

\section{Conclusion}

Chronic renal failure undergoing haemodialysis in not contraindication for chemotherapy, but indications for the use of chemotherapy in these patients should be considered carefully. We reported the case of patient with small-cell lung cancer and chronic 
Citation: Bursac D (2018) Safely Chemotherapy Administration in Patient with Small-cell Lung Cancer and End-stage Renal Disease Undergoing Haemodialysis. J Carcinog Mutagen 9: 324. doi:10.4172/2157-2518.1000324

Page 3 of 3

renal failure undergoing haemodialysis successfully treated with first and second line chemotherapy. Close cooperation between oncologist and nephrologist is essential to improve outcomes in these complex patients.

\section{References}

1. Eisenhauer EA, Therasse P, Bogaerts J, Schwartz LH, Sargent D, et al. (2009) New response evaluation criteria in solid tumours: Revised RECIST guideline (version 1.1). Eur J Cancer 45: 228-247.

2. Kim YH et al. (2010) Successful treatment with carboplatin and etoposide in a small-cell lung cancer patient undergoing haemodialysis. Respir Med 3: $68-70$.

3. Janus N, Thariat J, Boulanger H, Deray G, Launay-Vacher V (2010) Proposal for dosage adjustment and timing of chemotherapy in haemodialysis patients. Ann Oncol 21: 1395-1403.

4. Yanagawa H, Takishita Y, Bando H, Sumitani H, Okada S (2006) Carboplatin-based chemotherapy in patients undergoing haemodialysis. Anticancer Res 16: 533-535.
5. Velasco CJ, Munoz J, DeJesus M, Soman S, Wang D (2011) The outcome of end-stage renal disease patients of dialysis who undergo chemotherapy. J Clin Oncol e16593.

6. Inoue A, Saijo Y, Kikuchi T, Gomi K, Suzuki T, et al. (2004) Pharmacokinetic analysis of combination chemotherapy with carboplatin and etoposide in small-cell lung cancer patients undergoing haemodialysis. Ann Oncol 15: 51-54.

7. Pedrazzoli P, Silvestris N, Santoro A, Secondino S, Brunetti O, et al. (2017) Management of patients with end stage renal disease undergoing chemotherapy: recommendations of the Associazione Italiana di Oncologia Medica (AIOM) and the Società Italiana di Nefrologia (SIN). ESMO Open 2: e000167.

8. Funakoshi T, Horimatsu T, Nakamura M, Suyama K,Mizukami T, et al. (2016) Chemotherapy in cancer patients undergoing hemodialysis: A multicenter study. Ann Oncol 27: vi497-vi521. 